This is an electronic reprint of the original article. This reprint may differ from the original in pagination and typographic detail.

\author{
Author(s): Liu, Yang; Wang, Mei; Villberg, Jari; Torsheim, Torbjorn; Tynjälä, Jorma; Lv, Yan; \\ Kannas, Lasse
}

Title: $\quad$ Reliability and Validity of Family Affluence Scale (FAS II) among Adolescents in Beijing, China

Year: $\quad 2012$

Version:

Please cite the original version:

Liu, Y., Wang, M., Villberg, J., Torsheim, T., Tynjälä, J., Lv, Y., \& Kannas, L. (2012).

Reliability and Validity of Family Affluence Scale (FAS II) among Adolescents in

Beijing, China. Child Indicators Research, 5(2), 235-251.

https://doi.org/10.1007/s12187-011-9131-5

All material supplied via JYX is protected by copyright and other intellectual property rights, and duplication or sale of all or part of any of the repository collections is not permitted, except that material may be duplicated by you for your research use or educational purposes in electronic or print form. You must obtain permission for any other use. Electronic or print copies may not be offered, whether for sale or otherwise to anyone who is not an authorised user. 


\title{
Reliability and Validity of Family Affluence Scale (FAS II) among Adolescents in Beijing, China
}

Y ang Liu · M ei Wang · Jari V illberg · Torbjørn Torsheim · J orma Tynjälä · Y an $\mathrm{LV} \cdot \mathrm{L}$ asse $\mathrm{K}$ annas

Y. Liu* · J . Villberg $\cdot$ J. Tynjälä $\cdot$ L. Kannas

*Corresponding author

Research C entre for Health Promotion, Department of H ealth Sciences, U niversity of J yväskylä, P.O .Box 35 (Viveca), J yväskylä, 40014, Finland

e-mail: yang.liu@jyu.fi

M. Wang

Mass Research Centre, China Institute of Sport Science, Beijing, China

T. Torsheim

Department of P sychosocial Science, U niversity of Bergen, Bergen, Norway

Y. LV

Zhejiang Institute of Sports Science, Hangzhou, China

\begin{abstract}
A bstract This study comprises two sub-studies. Study I assessed the test-retest reliability of Family A ffluence Scale (FAS II) items among 95 students aged 11 and 15 years old in B eijing. Study II investigated the completion rate of traditional indicators (parents' educational level, perceived family wealth, resident area, and school location) measuring socioeconomic status (SES) compared with FAS II, and examined the internal reliability, external and construct validity of the FAS II items in a population of 5876 schoolchildren aged 11,13 and 15 years old in B eijing. Our study found that the FAS II items have high completion rates (>99\%) which are better than other SES indicators. A nalyses of reliability showed a moderate internal reliability (Cronbach's alpha $=0.58$ ) and at least substantial test-retest reliability $(I C C>0.75)$. M oderate external validity of FAS II was found by Spearman rank correlation between FAS II and other SES indicators (parental education level and perceived family wealth) $\left(r_{s}=0.48-0.51, p<0.001\right)$ and ordinal regressions. Graphical log-linear Rasch model (GLLRM ) showed that FAS has adequate construct validity (few LD and weak DIF). In conclusion, the FAS II is a reliable and valid SES measure for adolescents in the B eijing area.
\end{abstract}

Keywords Adolescents, F amily Affluence Scale, Health Behaviour in School-aged Children (HBSC), Reliability, Socioeconomic inequality, Validity 


\section{Introduction}

Social inequalities in health have been an important research topic in both social science and medicine (Dalstra et al. 2006; Marmot 2005). Research on the association of social inequalities and health behaviour also revealed that socioeconomic status plays a role in the explanation of the variant prevalence of the health behaviour (Ritterman et al. 2009). M oreover, health inequalities in young children and adults are well-established, but there has been debate about health inequalities in adolescents (West and Sweeting 2004). Inconsistent or nonassociations have been found between socioeconomic status (SES) and some health outcomes among adolescents (Goodman 1999; Goodman 2001; Starfield et al. 2002; W est 1997; W est and Sweeting 2004). Despite the genuine relationships of SES and health outcomes, the results and findings vary according to sample age groups and research methods, one possible reason for this is that various measures of SES were employed and therefore exert different effects on the same health outcome (Currie et al. 2008), and there might not be sufficient indicators to measure SES of adolescents specifically (B oyce et al. 2006). In the last two decades, the Chinese economy has grown rapidly, resulting in the achievement of improving people's health as well as improving health care. However, these health developments did not automatically follow the economic growth. In actuality, a widening gap in both health status and health care between different social economic statuses was found more often in China (Li and Zhu 2006; Liu et al. 1999). Thus, it is crucially important to develop reliable and valid indicators to measuring SES in China in order to get an accurate picture of the evidence-based results of socio-inequalities in health.

For an adult population, SES is traditionally assessed by education, occupation and income (Ostrove and A dler 1998). A dolescents, normally, are in the period of spending most of their time studying in school and not legally allowed to work, so they do not have any, or have very little economic resources themselves. Accordingly, adolescents' SES is usually measured by using the information of their parents' SES, such as parents' education and occupation, and household income. However, one should be aware of the difficulties of measuring adolescents' SES when using their parental SES as a proxy. As Currie and her colleagues (2008) argued conceptually, it is still uncertain whether parents' SES should be used as a proxy. For instance, the adolescents' SES are not always reflecting their parental SES, and even if it does so, which is the most relevant aspect of SES. Furthermore, methodologically there are also difficulties in obtaining accurate information regarding parental SES from adolescents due to the lack of knowledge or unwillingness to reveal the relevant information (Currie et al. 2008) which results in the low completion or high non-response rates when collecting data of adolescents self-reported parental SES (M olcho et al. 2007, $W$ ardle et al. 2002). In addition, it has been reported that there is a higher nonresponse rate for those adolescents in the low SES group (W ardle et al. 2004). Therefore, when measuring the SES of adolescents, it is crucially important to formulate accurate and effective indicators, which are easy for adolescents to answer and minimize the potential bias in a certain subgroup.

Among the indicators used for measuring adolescents' SES, the Health Behaviour in School-A ged Children (HBSC) Family A ffluence Scale (FAS) is a measure which has recently been developed and widely used to address the 
association of SES and health outcomes in both domestically and internationally comparative studies (Currie et al. 2008; M aes et al. 2006; Pickett et al. 2005; Torsheim et al. 2004; Torsheim et al. 2006). The FAS used in the HBSC survey contains several items which assess the SES of the adolescent based on the material condition of their household, which was originally based on the work of Carstairs and Morris (1991) and Townsend (1987). In the 1997/1998 HBSC survey, three FAS items were employed, which asked about family car ownership, the child's use of their own bedroom, and the number of family holidays, referred to as the first version of FAS (FAS I) (Currie et al. 2004). Later, in the 2001/2002 HBSC survey, an item on number of family computers was added and these four FAS items was named as the second version of FAS (FAS II) (Currie et al. 2004).

The FAS II has been used extensively in the HBSC study in the past decade to examine and describe socioeconomic inequalities in relation to adolescent health outcomes. Compared to the traditional SES indicators, one advantage of the FAS II items is that it can greatly reduce the non-response rate. It has been reported that the FAS II items showed a higher completion rate than items on parental education and occupation (Boudreau and Poulin 2009; Lin 2011; M olcho et al. 2007). Validation studies of the FAS II have been done in many HBSC member countries as well as some non-HBSC member countries, from which the results showed that the FAS II items have a moderate internal reliability (Lin 2011; Molcho et al. 2007; W ardle et al. 2002), a significant association with parental education and occupation (Currie et al. 1997; Molcho et al. 2007), a high agreement rate between adolescents and parents (Andersen et al. 2008), and a satisfactory correlation with the national wealth indicator, Gross Domestic Product (GDP) (Boyce et al. 2006). Although the test-retest reliability of FAS II items has not been examined, the rather higher agreement can be predicted due to the objectivity and stability of the indicators (Currie et al. 2008). Moreover, Schnohr and her colleagues (2008) have analyzed the differential item functioning (DIF) of FAS II and demonstrated that it can be used as an interval scale and a measure of wealth within a country and between countries.

The FAS II has also recently been used as a SES indicator outside the context of the HBSC study (e.g. West and Sweeting 2004) or in countries which are not a member of the HBSC network (e.g. Cho and K hang 2010). Because every country has its own culture and economic status, the FAS II should be used after examining the applicability and validity in a certain context. However, since the HBSC study is limited at the moment to countries in Europe and North A merica, few studies assessing the use of the FAS II have been done elsewhere in the world. To the authors' knowledge, there are only two studies which have been done recently to assess the use of FAS II in A sia (Cho and K hang 2010; Lin 2011) and no similar study has been done in the mainland China. The purposes of this study are, therefore, to investigate the completion rate of the traditional indicators measuring SES compared with FAS II, and to examine the reliability and validity of the FAS II items in a Chinese adolescent population.

\section{Methods}

This paper comprises data from two separate studies conducted by the China Institute of Sport Science (CISS) in the Beijing area, both following the HBSC research protocol (Currie et al. 2001). B eijing is the capital of China, consisting of 
16 districts and 2 counties, with the total population 16.95 million by the end of 2009. Beijing has 653255 students in 1104 primary schools and 508327 students in 634 secondary and upper secondary schools (Beijing Municipal Bureau of Statistics 2010). In 2010, the average annual income in Beijing was 29073 Chinese $Y$ uan (around $4419 \$$ ) per person, which is approximately 1.2 times the average annual income for China (3633\$) (B eijing M unicipal Bureau of Statistics 2010).

\subsection{Study I. Test-retest reliability study}

The data for test-retest reliability analysis was from the pilot study for the HBSC based behaviour and lifestyle survey for school-aged children in Beijing in October 2008. A sample of 95 male and female students aged 11 or 15 years old participated in a test and retest with a three weeks interval (the demographic characteristics of respondents can be seen in Table 1). Student identity numbers of respondents were utilized to permit matching of test-retest questionnaires. The test-retest reliability of FAS II items was estimated with the single measure of Intraclass Correlation Coefficients (ICC) (Shrout and Fleiss 1979) through case 2 (using a two-way random model with an absolute agreement type), with $95 \%$ confidence interval $(\mathrm{Cl})$. The detailed information of Study $\mathrm{I}$ is described elsewhere (Liu et al. 2010).

\subsection{Study II. Internal reliability and validity study}

\subsubsection{Study subjects}

The data for all the other analyses were from the HBSC based behaviour and lifestyle survey for school-aged children in Beijing, which was carried out between November and December 2008 by the China Institute of Sport Science (CISS). The survey sampled from state schools all over the Beijing area. The Chinese school and class systems are not widely known in western countries: sometimes students in the schools, and even in the classes of a school, are arranged according to geographic location, academic achievement etc. Therefore, in order to effectively choose the most representative sample, the survey sampling included three stages and two strata that introduced the detail as follows:

Stage I. Selection of the sample districts (counties)

The stratified random sample method was used in this stage. The first step of this stage was to stratify all the 16 districts and 2 counties of Beijing area according to geographical location. Two strata, urban area and rural area were stratified in this step. The second step was to stratify both the urban and rural areas in terms of social economic development level. Three strata, good, fair and poor, were used. The last step of this stage was to choose one district (county) randomly from each level in both urban and rural areas.

Stage II. Selection of the sample schools

The stratified random sample method was used in this stage. Firstly, all schools in each sample district (county) were divided into three categories on the basis of school conditions (good, fair and poor). Then, one primary school, one secondary school and one upper secondary school were 
selected randomly from each category in each sample district (county). In order to ensure the convenience of the survey, both the secondary school and the upper secondary school could be drawn from the same high school which includes both secondary and upper secondary school.

Stage III. Selection of the sample classes

The random sampling method or stratified random sampling method is used in this stage.

A total of 5985 schoolchildren were sampled following the above mentioned three stages. Samples were cleaned in line with the cleaning rule of the HBSC survey protocol if their gender or birth date were missing, or their age was beyond the target range (Currie et al. 2001). The final data used for analyses contained a total of 5876 schoolchildren, making a completion rate of $98.2 \%$ (the demographic characteristics of respondents can be seen from Table 1).

Table 1 D emographic characteristics of respondents in Study I and Study II

\begin{tabular}{ccclccc}
\hline & \multicolumn{3}{c}{ Sample size } & & \multicolumn{2}{c}{ Age } \\
\cline { 6 - 7 } \cline { 6 - 7 } & $n$ & $\%$ & & M ean age & SD \\
\hline Study I & & & & & \\
Total & 95 & 100.0 & & $/$ & $/$ \\
B oys & 51 & 53.7 & & $/$ & $/$ \\
Girls & 44 & 46.3 & & $/$ & $/$ \\
11yrs & 44 & 46.3 & & 11.70 & 0.35 \\
15yrs & 51 & 53.7 & & 15.80 & 0.32 \\
Study II & & & & & \\
Total & 5876 & 100.0 & & $/$ & $/$ \\
Boys & 2816 & 47.9 & & $/$ & $/$ \\
Girls & 3060 & 52.1 & & $/$ & $/$ \\
11yrs & 1941 & 33.0 & & 11.74 & 0.32 \\
13yrs & 1930 & 32.8 & & 13.73 & 0.34 \\
15yrs & 2005 & 34.1 & & 15.78 & 0.32 \\
\hline
\end{tabular}

\subsubsection{Survey procedure}

All students in the selected sample class were asked to complete a self-report questionnaire during a normal school class with a teacher and/or researcher administering. The students were first instructed how to fill in the questionnaire. Student's participation in the survey was voluntary and the questionnaire could only be accessed by researcher. Students were also informed that only the researcher will read their answers. The questionnaire used in the Chinese survey was based on the English version of the questionnaire used in the Finnish HBSC Survey in 2006. The questionnaire was firstly translated from English to Chinese by two researchers independently and re-translated from Chinese to English by other professional translators to check for any discrepancies. Both studies mentioned here were approved by the ethics committee of CISS and the Research Centre for $\mathrm{H}$ ealth Promotion at the U niversity of Jyväskylä. 


\subsubsection{Variables used in analysis}

Family Affluence Scale II

The FAS II was used as a measure of SES in this study. The items, response categories, codes and analyses strategy of FAS II used in the present study are the following:

- "Does your family own a car, van or truck?"

R esponse categories were: No $(=0)$; Y es, one $(=1)$; Y es, two or more $(=2)$.

- "Do you have your own bedroom for yourself?"

Response categories were: No $(=0)$; Y es $(=1)$.

- "During the past 12 months, how many times did you travel away on holiday with your family?"

Response categories were: Not at all $(=0)$; Once $(=1)$; Twice $(=2)$; M ore than twice (=3).

- "How many computers does your family own?"

Response categories were: None $(=0)$; One $(=1)$; Two $(=2)$; M ore than two (= 3).

According to Currie and her colleagues (2008), a composite FAS score was calculated for each respondent based on his or her answers to these four items. Following previous HBSC surveys, the two highest response categories (' 2 ' and ' 3 or more') of the last two items (holidays and computers) were combined. Three groups were categorized in terms of the composite FAS score, in which FAS Iow (score $=0-3$ ) indicated low affluence, FAS medium (score $=4,5$ ) indicated middle affluence, and FAS high (score $=6,7)$ indicated high affluence.

Parents' educational level

Students were asked to choose their father's and mother's highest level of education from 7 alternatives, which are under primary school, primary school, secondary school, upper secondary school, junior college, university (bachelor level), and graduate or more. During the analyses, the parents' education were divided into three categories, low education level (under the primary school, primary school, secondary school), medium education level (upper second school, junior college), and high education level (university, graduate or more), for father and mother respectively.

Perceived Family Wealth

Perceived socioeconomic status of the household was enquired by asking the students' own perception of their family financial situation. The responses were very well off financially, quite well off financially, average, not very well off financially and not at all well off financially. The indicator of perceived family wealth was divided into three categories, perceived low family weal th (not very well off, not well off at all), perceived medium family wealth (average), and perceived high family wealth (very well off, quite well off).

U rban and rural

According to the sampling procedure, the information of selected schools in the survey can be retrieved, and the respondents were divided into urban and rural according to the school position. 


\section{School location}

The respondents were categorized into three SES groups, good, fair and poor, according to their school's location in districts with different socioeconomic development (B eijing M unicipal Bureau of Statistics 2010).

\subsubsection{Statistical analyses}

The analyses have been done to address the following aspects of the data according to the purpose of the study:

I tem completion rate

Completion rate was computed for FASII items and total FAS scores as well as the other two SES indicators (parental educational level and perceived family weal th). Chi-square test was used to compare the completion rate of FAS items by gender and by age groups as well as the completion rate among different SES measures.

Reliability

The internal consistency of the FAS II items and item-rest coefficient were examined with the Cronbach's alpha coefficient. Spearman correlations were used to assess the association between individual FAS II item, the total FAS scores and FAS category. In order to examine whether the four FAS II items are to reflect a general underlying dimension of affluence, the fit of a one-factor solution was tested with confirmatory factor analysis (CFA) for categorical data using the Robust Weighted Least Square (WLSMV) estimator. The primary fit indices included the Comparative Fit Index (CFI) and the weighted root mean square residual (WRM R), as recommended in recent publications (Finney and DiStefano 2006). A CFI e 0.95 and W RM R < 1 indicates a good fit to the data.

\section{Validity}

Spearman correlations were used to assess the association between the total FAS scores with parental educational level and perceived family wealth. Two ordinal logistic regression models were used for analyzing the association between FAS category and other SES indicators. The unadjusted odds ratio (OR) from model 1 (univariate ordinal regression model) and the adjusted OR from model 2 (included all independent variables) were computed respectively. Construct validity was investigated with the Graphical Log Linear Rasch Model (GLLRM) with Differential Item Function (DIF) and Local Dependence (LD).

All data of the present study were entered by Epidata 3.1 with double entry and validation. The analyses of item completion rate, reliability and validity were done by Predictive Analytics Software (PASW, formerly SPSS), version 18.0 (SPSS, Inc., Chicago, IL, US). The CFA was analyzed by M Plus 6.1 (M uthén and Muthén 2010). The GLLRM was performed by DIGRAM 2.0 (K reiner 2009). A $P$-value which was lower than 0.05 or $95 \%$ confidence intervals did not overlap was considered significantly different. 


\section{Results}

\subsection{Study I}

\section{Test-retest reliability}

Table 2 reveals the values of ICC for all respondents by gender and age. Overall, the values of ICC of the FAS II items, FAS score and FAS category ranged from 0.76 to 0.95 , with the lowest value for the item regarding family holidays, and the highest value for the item asking about own bedroom. A ccording to Landis and K och divisions of agreement (1977), all four items of FAS II as well as the FAS score and category showed at least substantial test-retest reliability. Gender and age differences of the agreement were only found for the item regarding own bedroom.

Table 2 ICC values for FA S II items, FA S score and FA S category by gender and age from Study I ( $\mathrm{N}=95)$

\begin{tabular}{|c|c|c|c|c|c|c|c|c|c|c|}
\hline & \multicolumn{2}{|c|}{ All $(\mathrm{N}=95)$} & \multicolumn{2}{|c|}{ B oys $(n=51)$} & \multicolumn{2}{|c|}{ Girls $(n=44)$} & \multicolumn{2}{|c|}{11 yrs $(n=44)$} & \multicolumn{2}{|c|}{15 yrs $(n=51)$} \\
\hline & ICC & $95 \% \mathrm{Cl}$ & ICC & $95 \% \mathrm{Cl}$ & ICC & $95 \% \mathrm{Cl}$ & ICC & $95 \% \mathrm{Cl}$ & ICC & $95 \% \mathrm{Cl}$ \\
\hline Family car & 0.88 & $0.83-0.92$ & 0.85 & $0.75-0.91$ & 0.92 & $0.86-0.96$ & 0.81 & $0.68-0.89$ & 0.92 & $0.86-0.95$ \\
\hline Own bedroom & 0.95 & $0.93-0.97$ & 1.00 & 1 & $0.92 *$ & $0.86-0.96$ & 0.79 & $0.65-0.88$ & $1.00^{\dagger}$ & 1 \\
\hline Family holidays & 0.76 & $0.66-0.83$ & 0.72 & $0.55-0.83$ & 0.77 & $0.61-0.87$ & 0.78 & $0.64-0.88$ & 0.64 & $0.44-0.79$ \\
\hline No. of computers & 0.77 & $0.67-0.84$ & 0.77 & $0.63-0.86$ & 0.76 & $0.61-0.86$ & 0.73 & $0.56-0.84$ & 0.79 & $0.66-0.87$ \\
\hline FAS score & 0.88 & $0.83-0.92$ & 0.85 & $0.75-0.91$ & 0.92 & $0.84-0.96$ & 0.83 & $0.70-0.90$ & 0.88 & $0.80-0.93$ \\
\hline FAS category & 0.78 & $0.69-0.85$ & 0.73 & $0.57-0.84$ & 0.84 & $0.72-0.91$ & 0.81 & $0.67-0.89$ & 0.68 & $0.50-0.80$ \\
\hline
\end{tabular}

\subsection{Study II}

\subsubsection{Completion rate}

In general, all items measuring SES in our study have a completion rate higher than $97 \%$. Out of 5876 pupils, the first and second lowest completion rate were items for parental education (father $97.9 \%$ and mother $98.4 \%$ ) and all the four items of FAS II have the same highest completion rate $(99.7 \%)$, which made the completion rate of the composite FAS scores $99.2 \%$. However, no significant differences were found between the completion rates of the four FAS II items and two parental education items. We also examined the completion rates by gender and by age groups. There is no difference in the completion rates of those SES items by gender except for the question asking about the family holidays ( $p=$ 0.042) which showed that boys were slightly less likely to report their family holidays than girls. Significant differences of completion rates were found in the three age groups for the item about asking father's education $(p<0.001)$ and mother's education ( $p<0.001$ ), which indicated that younger children were less likely to report their parental educational level. 


\subsubsection{Internal reliability}

As seen in Table 3, we examined the internal consistency of FAS II items and found a moderate association $( \pm=0.58$ ). The alpha coefficient changed if single item was deleted from the scale. The item-rest coefficients suggested that deleting either family car $( \pm=0.47)$, or family holidays $( \pm=0.49)$, or numbers of computer (0.43) items resulted in a drop of internal consistency. On the contrary, if own bedroom was deleted from the FAS II items, the alpha coefficient would improve to 0.60 . Higher internal consistencies were observed in older respondents.

Table 3 Cronbach A I pha coefficient for FAS II items by gender and age from Study II ( $\mathrm{N}=5876)$

\begin{tabular}{lrlcccc}
\hline & \multicolumn{6}{c}{ Cronbach A lpha } \\
\cline { 2 - 7 } & Total & Boys & Girls & $11 y r s$ & $13 y r s$ & $15 y r s$ \\
\hline All four items & 0.58 & 0.59 & 0.57 & 0.56 & 0.56 & 0.62 \\
Delete family car & 0.47 & 0.49 & 0.45 & 0.48 & 0.46 & 0.49 \\
Delete own bedroom & 0.60 & 0.61 & 0.59 & 0.55 & 0.57 & 0.66 \\
Delete family holiday & 0.49 & 0.50 & 0.48 & 0.49 & 0.45 & 0.53 \\
Delete N 0. Of computers & 0.43 & 0.43 & 0.42 & 0.40 & 0.42 & 0.47 \\
\hline
\end{tabular}

The results of the inter-correlations between the FAS II items and the composite FAS score showed moderate associations (more than 0.60) except for the item asking about own bedroom (0.34) (Table 4). Rather poor correlations were found within the FAS II items (from 0.16 to 0.39 ) and the poorest one was found between items asking about own bedroom and family car (0.16), but all items were intercorrelated.

Table 4 Spearman correlations of FAS II items from Study II (N =5876)

\begin{tabular}{lcccc}
\hline & $\begin{array}{c}\text { Family } \\
\text { car }\end{array}$ & $\begin{array}{c}\text { Own } \\
\text { bedroom }\end{array}$ & $\begin{array}{c}\text { Family } \\
\text { holidays }\end{array}$ & $\begin{array}{c}\text { No. Of } \\
\text { computers }\end{array}$ \\
\hline Own bedroom & $0.16^{* *}$ & & & \\
Family vacations & $0.32 * *$ & $0.22 * *$ & & \\
N o. of computers & $0.39 * *$ & $0.20 * *$ & $0.36 * *$ & \\
FA S score & $0.67 * *$ & $0.34 * *$ & $0.69 * *$ & $0.69 * *$ \\
\hline$*$ C orrelation is significant at the 0.01 level (2-tailed).
\end{tabular}

The CFA analyzed by MPlus showed that the one-factor model fitted the data relatively well as indicated by the comparative fit index (CFI) of 0.99 and the weighted root mean square residual of 1.10. The standardised factor loading ranged between 0.49 (number of own bedroom) and 0.69 (number of computers).

\subsubsection{Validity}

The external validity of FAS II was evaluated by examining associations between FAS II and other SES indicators, using Spearman rank correlation $\left(r_{s}\right)$, univariate and multivariate ordinal logistic regression analyses. Moderate associations were found between the composite FAS II scores with father's education $\left(r_{s}=0.49, p<\right.$ $0.001)$, mother's education $\left(r_{s}=0.48, p<0.001\right)$, and perceived family wealth $\left(r_{s}\right.$ $=0.51, p<0.001$ ). 
Table $\mathbf{5}$ The distribution of variables by FAS category and ordinal regression results, FA S low as the referent $(\mathrm{N}=5876)$

\begin{tabular}{|c|c|c|c|c|c|c|c|c|c|c|c|}
\hline & \multirow{2}{*}{$\frac{\text { Total }}{\mathrm{n}}$} & \multicolumn{2}{|c|}{$\begin{array}{l}\text { FAS } \\
\text { (low) }\end{array}$} & \multicolumn{2}{|c|}{$\begin{array}{c}\text { FAS } \\
\text { (middle) }\end{array}$} & \multicolumn{2}{|c|}{$\begin{array}{c}\text { FAS } \\
\text { (high) }\end{array}$} & \multicolumn{2}{|c|}{ Univariate OLR } & \multicolumn{2}{|c|}{ M ultivariate $\mathrm{OLR}$} \\
\hline & & $\mathrm{n}$ & $\%$ & $\mathrm{n}$ & $\%$ & $\mathrm{n}$ & $\%$ & OR & $95 \% \mathrm{Cl}$ & OR & $95 \% \mathrm{Cl}$ \\
\hline Overall & 5876 & 2421 & 41.2 & 2178 & 37.1 & 1229 & 20.9 & & & & \\
\hline \multicolumn{12}{|l|}{ Gender } \\
\hline Boy & 2816 & 1116 & 39.6 & 1015 & 36.0 & 655 & 23.3 & $0.84 * * *$ & $0.77-0.93$ & $0.82 * *$ & $0.79-0.88$ \\
\hline Girl & 3060 & 1305 & 42.6 & 1163 & 38.0 & 574 & 18.8 & 1.00 & & 1.00 & \\
\hline \multicolumn{12}{|l|}{ Age } \\
\hline 11 & 1941 & 624 & 32.2 & 776 & 40.0 & 525 & 27.0 & $0.48^{* * *}$ & $0.43-0.54$ & $0.54 * * *$ & $0.48-0.62$ \\
\hline 13 & 1930 & 779 & 40.4 & 753 & 39.0 & 373 & 19.3 & $0.70 * * *$ & $0.63-0.79$ & $0.67 * * *$ & $0.59-0.77$ \\
\hline 15 & 2005 & 1018 & 50.8 & 649 & 32.4 & 331 & 16.5 & 1.00 & & 1.00 & \\
\hline \multicolumn{12}{|l|}{ Area } \\
\hline Urban & 2833 & 833 & 29.4 & 1136 & 40.1 & 843 & 29.8 & $0.37 * * *$ & $0.33-0.40$ & $0.75^{* * *}$ & $0.64-0.84$ \\
\hline Rural & 3043 & 1588 & 52.2 & 1042 & 34.2 & 386 & 12.7 & 1.00 & & 1.00 & \\
\hline \multicolumn{12}{|c|}{ School location } \\
\hline Level 1 & 1943 & 626 & 32.2 & 750 & 38.6 & 555 & 28.6 & $0.39 * * *$ & $0.35-0.44$ & $0.57^{* * *}$ & $0.49-0.66$ \\
\hline Level 2 & 1956 & 731 & 37.4 & 798 & 40.8 & 409 & 20.9 & $0.53^{* * *}$ & $0.47-0.60$ & $0.62 * * *$ & $0.54-0.72$ \\
\hline Level 3 & 1977 & 1064 & 53.8 & 630 & 31.9 & 265 & 13.4 & 1.00 & & 1.00 & \\
\hline \multicolumn{12}{|c|}{ F ather's education } \\
\hline Low & 2017 & 1329 & 65.9 & 539 & 26.7 & 130 & 6.4 & $11.87^{* * *}$ & $10.29-13.71$ & $3.05^{* * *}$ & $2.46-3.78$ \\
\hline Middle & 2278 & 845 & 37.1 & 968 & 42.5 & 446 & 19.6 & $3.48 * * *$ & $3.06-3.96$ & $1.66^{* * *}$ & $1.40-1.95$ \\
\hline High & 1456 & 187 & 12.8 & 624 & 42.9 & 637 & 43.8 & 1.00 & & 1.00 & \\
\hline
\end{tabular}

**overall effect of the variable in the model $p<0.01$,

$* * *$ overall effect of the variable in the model $p<0.001$ 
Table 5 continued

\begin{tabular}{|c|c|c|c|c|c|c|c|c|c|c|c|}
\hline & \multirow{2}{*}{$\frac{\text { Total }}{n}$} & \multicolumn{2}{|c|}{$\begin{array}{l}\text { FAS } \\
\text { (low) }\end{array}$} & \multicolumn{2}{|c|}{$\begin{array}{c}\text { FAS } \\
\text { (middle) }\end{array}$} & \multicolumn{2}{|c|}{$\begin{array}{c}\text { FAS } \\
\text { (high) }\end{array}$} & \multicolumn{2}{|c|}{ Univariate OLR } & \multicolumn{2}{|c|}{ M ultivariate OLR } \\
\hline & & $\mathrm{n}$ & $\%$ & $\mathrm{n}$ & $\%$ & $\mathrm{n}$ & $\%$ & OR & $95 \% \mathrm{Cl}$ & OR & $95 \% \mathrm{Cl}$ \\
\hline \multicolumn{12}{|c|}{ M other's education } \\
\hline Low & 2154 & 1384 & 64.3 & 600 & 27.9 & 146 & 6.8 & $11.31 * * *$ & $9.80-13.07$ & $2.54 * * *$ & $2.05-3.15$ \\
\hline M iddle & 2301 & 818 & 35.5 & 999 & 43.4 & 470 & 20.4 & $3.34 * * *$ & 2.93-3.81 & $1.55^{* * *}$ & $1.31-1.83$ \\
\hline High & 1327 & 177 & 13.3 & 545 & 41.1 & 597 & 45.0 & 1.00 & & 1.00 & \\
\hline \multicolumn{12}{|c|}{ Perceive family wealth } \\
\hline Low & 752 & 605 & 80.5 & 113 & 15.0 & 27 & 3.6 & $21.43 * * *$ & $17.44-26.34$ & $10.51 * * *$ & $8.44-13.12$ \\
\hline M iddle & 2918 & 1444 & 49.5 & 1126 & 38.6 & 325 & 11.1 & $5.02 * * *$ & 4.49-5.62 & $3.25^{* * *}$ & $2.88-3.67$ \\
\hline High & 2185 & 369 & 16.9 & 930 & 42.6 & 870 & 39.8 & 1.00 & & 1.00 & \\
\hline
\end{tabular}

*** overall effect of the variable in the model $p<0.001$ 
Table 5 shows the distribution of all variables used in this study by FAS category and the unadjusted and adjusted odds ratios (OR's) for all independent variables, with FAS low as the referent group. Students in rural areas were more likely to be located in the low FAS group. The percentage of respondents in high FAS group increased if they reported their father or mother has a higher educational background. Similarly, the percentage of students in high FAS group decreased with their perceived family wealth. Both models revealed the same significant association between FAS II category and all independent variables (Table 5). $O R$ 's for gender and age showed that girls and elder students were more likely to report less family affluence, compared to boys and young students. Similar findings were also established for students who lived in rural areas or studied in a school from the district with poor economic status. The multivariate analyses indicated that students whose fathers' educational level was low and middle were respectively around 3 times and 1.7 times more likely to report less affluence than students whose fathers' educational level were high. Similar results were also observed for respondents with low and middle level educational mothers, where the OR's were about 2 and 1.6 respectively. The most prominent finding was that students whose perceived family wealth was low were 10 times more likely to report their family affluence was also low, compared to those who perceived their family wealth as high.

To highlight the construct validity of the indicators, the GLLRM model fitting item responses for our data is shown in Figure 1. Two coefficients were significant revealing LD (Local Dependence) between items; number of carsnumber of computers $\left(^{3^{p}}=0.15\right)$ and number of cars-number of holidays $\left(^{3^{p}}=-\right.$ 0.13 ), which meant that there were correlation between the number of cars and number of computers, as well as the number of cars and number of holidays. However, these correlations are weak. The DIF (Differential Item Functioning) was observed between age and three items, 'own bedroom', 'number of holidays', and 'number of computers'. The results indicate that the chances of having own bedroom $\left(3^{p}=0.41\right)$ and number of computers $\left(3^{p}=0.16\right)$ increased with age, whereas the number of holidays $\left({ }^{\mathrm{P}}=-0.28\right)$ decreased with age. The DIF was not found between gender and FAS II items.

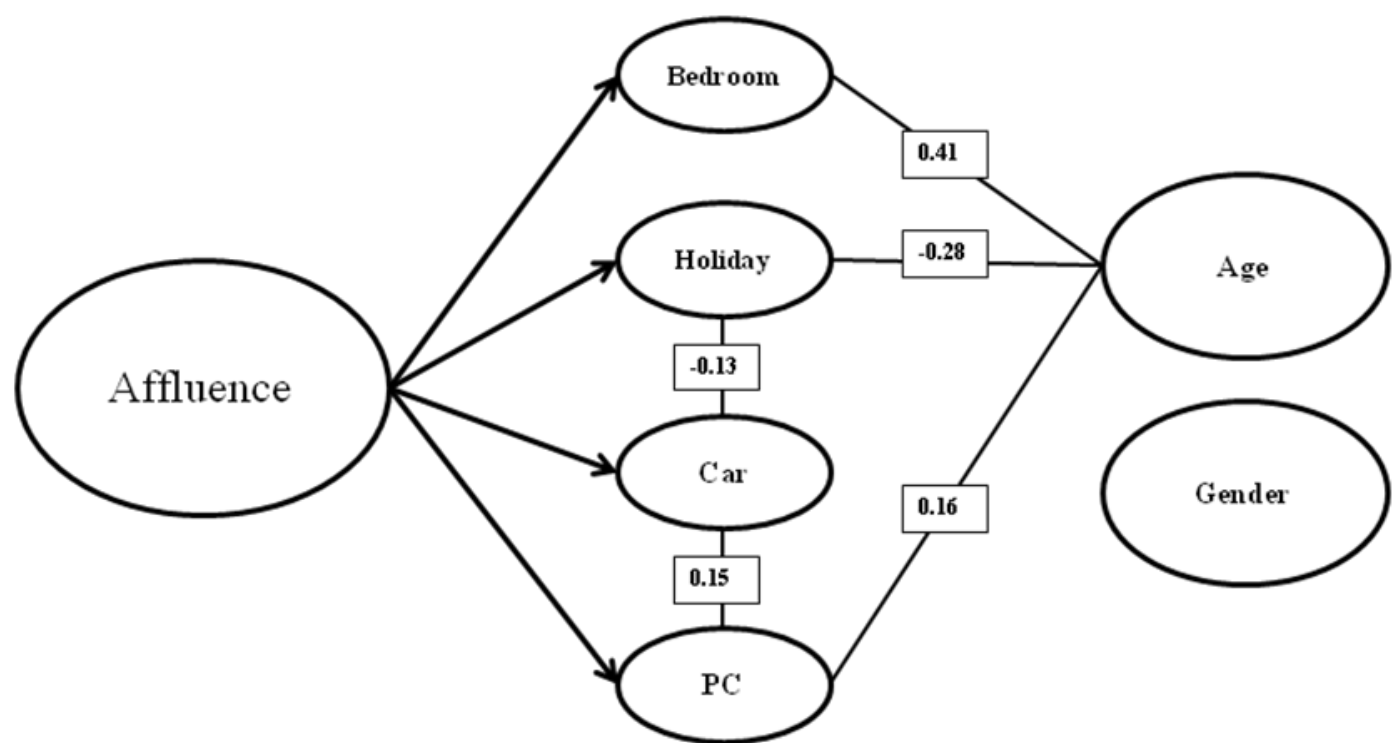

Figure 1 The generalization of FAS II to GLLRM (number in boxes are partial gamma coefficients, ${ }^{3 \mathrm{P}}$, indicate a significant correlation beyond the latent trait) from Study II (N $=5876)$. 


\section{Discussion and conclusion}

To obtain the accurate finding of social inequalities in health, it is critical to employ reliable and valid indicators which measure socioeconomic status. Although FAS II, widely used in HBSC network countries in Europe and North A merica, was proved to be an easily answered and a useful indicator of child material affluence, it should be examined whether FAS II is applicable before it is used in countries other than the current HBSC countries. As the first study to assess the usability of FAS II in a Chinese context, in general, we found that of the FAS II items have high completion rates, a moderate internal reliability, at least substantial test-retest reliability, moderate associations with other SES indicators, only few LD and weak DIF. This indicates that the FAS II is a useful and valid SES measure for adolescents in the Beijing area.

Previous studies demonstrated the difficulties of obtaining information on parental education, occupation, and income, which are usually used as SES markers in adolescent studies and it has been reported in many studies that there is a high non-response rate for those questions (Cho and K hang 2010; Currie et al. 1997; Lin 2011; M olcho et al. 2007; Wardle et al. 2002). In contrast, it has been highlighted in previous studies that the FAS II items have much higher completion rates than other traditional SES indicators (Cho and K hang 2010; Lin 2011; M olcho et al. 2007; Wardle et al. 2002). In our study, therefore, it is not surprising that a higher proportion of the respondents answered the four FAS II items $(99.7 \%)$ than items on parents' highest educational level $(97.9 \%$ for father and $98.4 \%$ for mother). However, the completion rates for parental education questions in our study were much higher than previous studies, although they revealed the lowest completion rates among all SES indicators analyzed in the present study. One possible reason might be that students were instructed to answer all the questions in sequence at the beginning of the survey which generated very low non-response rate for all survey questions. Consequently, no significant difference was found between the completion rates of the four FAS II items and the other SES indicators. When comparing the completion rates of the SES measures across gender and age, we found that there was no gender difference, which is similar to the results reported by Molcho and his colleagues (2007) except for the question asking about the family holidays $(p=0.042)$. A ge differences were found for items asking about parental education $(p<0.001)$, indicating that younger children were less likely to report their parental educational level, again, similar results were also found by M olcho et al (2007) and Lin (2011). W e also found age differences of two FA S II items, family car ( $p$ $=0.009$ ) and own bedroom $(p=0.001)$, which are not consistent with other studies (Lin 2011; M olcho et al. 2007).

Concerning the reliability of FAS II, in our study, the internal correlations between the FAS II items were low but all items were intercorrelated $\left(r_{s}=0.16\right.$ $0.39, p<0.001)$ and the Cronbach's alpha was moderate $(0.58)$, which is in line with previous studies (Cho and Khang 2010; Lin 2011; Molcho et al. 2007; Schnohr et al. 2008). These results were understandable due to the limited range of the alternatives and each item describing a different aspect of family affluence (W ardle et al. 2002). Similar to earlier studies (Boudreau and Poulin 2009; Cho and K hang 2010; Lin 2011), we found that the item on own bedroom had weaker correlations with the other FAS II items and the composite FA S score. In addition, the item-rest coefficients in our study concluded that if the item on own bedroom 
was deleted from the FAS scale, the alpha coefficient would improve whereas deleting any other of the three items would reduce the internal consistency. Those findings suggested that further examination should be considered to choose the most sensitive items in specific settings since country and culture difference may affect the relative contribution of the four FAS II items to the composite FAS II (Schnohr et al. 2008). One extra item which may be considered and tested in the future is that to ask the amount of weekly pocket money received by the student, which the child will reliably know the answer to, and is related to family affluence to some extent.

The significant association between FA S II and other traditional SES measures, such as parental education and/or parental occupation (Lin 2011; M olcho et al. 2007; Richter et al. 2009) were found in previous studies, although the strength of associations varied in different countries. The rather low strength of association found in the present study was accounted for by the fact that FAS and parental education and occupation examine different aspects of socioeconomic status per se, and therefore may not overlap (M olcho et al. 2007; W ardle et al. 2002). Our study revealed moderate associations between FAS and other SES indicators. The strength of associations in the present study $\left(r_{s}=0.48-0.51, p<0.001\right)$ were higher than previous findings possibly indicating that the FAS II was related to other SES measures more closely in a Chinese context than others. We also assessed the external validity of FAS II by ordinal logistic regression and the results were in line with the correlation analyses which confirmed the significant association between FAS II and other SES indicators. For instance, the students who perceived their family wealth as low were 10 times more likely to be in the Iow FAS category than those who perceived their family wealth as high, suggesting that the FAS II was more likely measuring the similar dimension of SES as perceived family wealth. This finding presented the possibility of adding the item on perceived family wealth as one item of the FAS scale for the development of the family affluences measure in the future, in line with Schnohr and her colleagues' (2008), who have purposed that the FAS II can be used as a measure of wealth within a country and between countries, plus the item on perceived family weal th was included. However, we should notice that perceived family wealth is a subjective concept, and therefore it may not be comparable to measures of the FAS II which are more concrete in nature. Furthermore, the results of GLLRM model presented in this study indicated that the FAS II items are nearly locally independent and have very weak DIF, which can be considered as satisfied construct validity according to R osenbaum (1989).

The strength of the present study is that we examined the test-retest reliability of FAS II, which has not been done and reported in earlier studies. A s expected by Currie and her colleagues (2008), the results revealed that all four FAS II items and the composite FAS score and category had at least substantial test-retest reliability due to the objectivity of the measures. M oreover, it is the first study to examine the cross cultural application of the FAS II comprehensively using a large sample of Chinese adolescents. At the same time, the current study still has several limitations. First of all, the data were only sampled from the B eijing area due to the aims of the survey and the limited resources. As a country with huge socioeconomic difference such as China, it may raise the bias to determine the usability of the FAS II if the sample cannot represent the whole nation. A nother major limitation is that the data were retrieved from self-report questionnaires from the adolescents and there is a lack of objective measures of respondents' family wealth, for instance, investigating the financial circumstances of the 
parents. In order to examine and develop the FAS II as a SES measure in China, more research with other objective measures should be encouraged in more and wilder locations.

In conclusion, as the first study on the reliability and validity of the HBSC FAS II in an adolescent population in Beijing, the present study showed that the FAS II had a high completion rate, better than other SES indicators. M oderate internal reliability and at least substantial test-retest reliability, as well as adequate external and construct validity, were found for the FAS II. In conclusion, the FAS II is a useful and valid SES measure for adolescents in the B eijing area.

Acknowledgements The authors would like to thank M s. L anmin Xiao from B eijing Experimental School of Xicheng District and M s. Jing Tian from B eijing A cademy of Educational Sciences for hel ping to organize and conduct the field work. The authors also would like to thank M r. M ichael Ormshaw for checking the language of the manuscript. As a part of joint research project, this study was supported by the China Institute of Sport Science (CISS) and the Research Centre for Health Promotion at the Department of H ealth Science in the U niversity of J yväskylä. The survey and data collection were funded by the CISS. D uring this research, the first author was supported by grants from the Finnish Foundation for A Icohol Studies and the U niversity of J yväskylä.

\section{References}

A ndersen, A., Krølner, R., Currie, C., Dallago, L., Due, P., Richter, M ., Orkényi, A ., \& H olstein, B. E. (2008). High agreement on family affluence between children's and parents' reports: international study of 11 -year-old children. J ournal of E pidemiology and Community $\mathrm{H}$ ealth, 62, 1092-1094.

B eijing M unicipal B ureau of Statistics. (2010). B eijing statistical year book. B eijing: China Statistical Publishing House.

B oudreau, B., \& Poulin, C. (2009). A n examination of the validity of the Family Affluence Scale II (FAS II) in a general adolescent population of Canada. Social Indicators Research, 94, 2942.

B oyce, W ., Torsheim, T., Currie, C., \& Zambon, A . (2006). The Family A ffluence Scale as a measure of national wealth: $V$ alidation of an adolescent self-report measure. Social Indicators Research, 78, 473-487.

Cantril, H. (1965). The pattern of human concern. N ew B runswick, NJ : Rutgers U niversity Press.

Carstairs, V., \& M orris, R. (1991). Deprivation and health in Scotland. A berdeen: A berdeen University Press.

Cho, H. J., \& K hang, Y. H. (2010). Family Affluence Scale, other socioeconomic position indicators, and self-rated health among South K orean adolescents: Findings from the K orea Y outh Risk Behavior W eb-based Survey (KY RBWS). J ournal of Public Health, 18(2), 169178.

Currie, C., Elton, R. A ., Todd, J., \& Platt, S. (1997). Indicators of socioeconomic status for adolescents: the WHO Health Behaviour in School-aged Children Survey. Health Education Research, 12(3), 385-397.

Currie, C., M olcho, M., B oyce, W., H olstein, B. E., Torsheim, T., \& Richter, M . (2008). Researching health inequalities in adolescents: The development of the $H$ ealth $B$ ehaviour in School-A ged Children (HBSC) Family A ffluence Scale. Social Science \& M edicine, 66, 1429-1436.

Currie, C., Nic Gabhainn, S., Godeau, E., Roberts, C., Smith, R., Currie, D., Pickett, W ., M organ, A ., \& B arnekow, V. (Eds.), (2008). Inequal ities in young people's health: HBSC international report from the 2005/2006 survey. Copenhagen, Denmark: WHO Regional Office for Europe.

Currie, C., Roberts, C., M organ, A., Smith, R., Settertobulte, W., Samdal, O., \& Barnekow, V. (Eds.), (2004). Y oung people's health in context. Copenhagen, Denmark: W HO Regional Office for Europe. 
Currie, C., Samdal, O., B oyce, W., \& Smith, R. (Eds.), (2001). Health Behaviour in Schoolaged Children: a WHO Cross-National Study (HBSC), Research Protocol for the 2001/2002 Survey. Edinburgh: Child and A dolescent Health Research U nit (CA HRU), U niversity of Edinburgh.

Dalstra, J., K unst, A ., \& M ackenbach, J. (2006). A comparative appraisal of the relationship of education, income and housing tenure with less than good health among the elderly in Europe. Social Science \& M edicine, 62, 2046-2060.

Goodman, E. (1999). The role of socioeconomic status gradients in explaining differences in US adolescents' health. A merican J ournal of Public Health, 89, 1522-1528.

Goodman, E., A dler, N. E., Kawachi, I., Frazier, A. L., Huang, B., \& Colditz, G. A . (2001). A dolescents' perceptions of social status: Development and evaluation of a new indicator. Pediatrics, 108(2), 1-8.

Finney, S. J., \& DiStefano, C. (2006). N on-normal and categorical data in structural equation modeling. In G.R. Hancock \& R.O. M ueller (Eds.), Structural equation modeling: A second course. Greenwich, CT: Information A ge Publishing.

K reiner, S. (2009). A guided tour through DIGRAM 2.0: A nalysis of contingency tables by chain graph models. Copenhagen: Department of B iostatistics, University of Copenhagen.

Landis, J. R., \& K och, G. G. (1977). The measurement of observer agreement for categorical data. Biometrics, 33, 159-174.

Li, H., Zhu, Y . (2006). Income, income inequality, and health: Evidence from China. J ournal of Comparative Economics, 34, 668-693.

Lin, Y . C. (2011). A ssessing the use of the Family A ffluence Scale as socioeconomic indicators for researching health inequalities in Taiwan adolescents. Social Indicators Research, 102, 463-475.

Liu, Y ., H siao, W ., \& Eggleston, K. (1999). Equity in health and health care: the Chinese experience. Social Science \& M edicine, 49, 1349-1356.

Liu, Y ., W ang, M ., Tynjälä, J ., L v, Y ., V illberg, J ., Zhang, Z., \& K annas, L. (2010). Test-retest reliability of selected items of Health B ehaviour in School-aged Children (HBSC) survey questionnaire in Beijing, China. B M C M edical Research M ethodology, 10:73.

M aes, L., V ereecken, C., V anobbergen, J., \& H onkala, S. (2006). Tooth brushing and social characteristics of families in 32 countries. International Dental J ournal, 56, 159-167.

M armot, M . (2005). Social determinants of health inequalities. The L ancet, 365, 1099-1104.

M olcho, M., Nic Gabhainn, S., \& K elleher, C. (2007). A ssessing the use of the Family Affluence Scale (FAS) among Irish schoolchildren. Irish M edical J ournal, 100(8), 37-39.

M uthén, L. K ., \& M uthén, B . O. (2010). M plus U ser's Guide, 6th edn. Los A ngeles, CA : M uthén $\&$ M uthén.

Ostrove, J. M ., \& A dler, N. E. (1998). Socioeconomic status and health. Current Opinion in Psychiatry, 11, 649-653.

Pickett, W., M olcho, M ., Simpson, K ., J anssen, I., K untsche, E., M azur, J., Harel, Y ., \& B oyce, W. (2005). Cross-national study of injury and social determinants in adolescents. Injury Prevention, 11, 213-218.

Ritterman, M . L., Fernald, L. C., Ozer, E. J ., A dler, N. E., Gutierrez, J. P., \& Syme, S. L. (2009). $O$ bjective and subjective social class gradients for substance use among $M$ exican adolescents. Social Science \& M edicine, 68, 1843-1851.

Rosenbaum, P. R. (1989). Criterion-related construct validity. Psychometrika, 54, 625-633.

Schnohr, C., K reiner, S., Due, P., Currie, C., B oyce, W., \& Diderichsen, F. (2008). Differential item functioning of a family affluence scale: V alidation study on data from HBSC 2001/02. Social Indicators Research, 89(1), 79-95.

Shrout, P. E., \& Fleiss, J. L. (1979). Intraclass correlations: use in assessing rater reliability. Psychological Bulletin, 86(2), 420-428.

Starfield, B ., Riley, A. W., Witt, W. P., \& Robertson, J . (2002). Social class gradients in health during adolescence. Journal of Epidemiology and Community Health, 56, 354-361.

Torsheim, T., Currie, C., B oyce, W., K al nins, I., Overpeck, M ., \& H aughland, S. (2004). M aterial deprivation and self-rated health: a multilevel study of adolescents from 22 European and $N$ orth A merican countries. Social Science \& M edicine, 59(1), 1-12.

Torsheim, T., Currie, C., B oyce, W., \& Samdal, O. (2006). Country material distribution and adolescents' perceived health: multilevel study of adolescents in 27 countries. J ournal of Epidemiology and Community Health, 60, 156-161.

Torsheim, T., Valimaa, R., \& Danielson, M . (2004). Health and well-being. In Currie C, Roberts C, M organ A, Smith R, Settertobulte W, Samdal O, Barnekow Rasmussen V. (Eds.), Y oung people's health in context: Health Policy for children and adolescents no. 4 (pp. 55-62). Copenhagen, Denmark: WHO Regional Office for Europe. 
Townsend, P. (1987). Deprivation. J ournal of Social Policy, 16(2), 147-164.

Wardle, J., Robb, K., \& J ohnson, F. (2002). A ssessing socioeconomic status in adolescents: the validity of a home affluence scale. J ournal of Epidemiology and Community Health, 56, 595599.

Wardle, J., Robb, K. A., J ohnson, F., Griffith, J., B runner, E., Power, C., \& M artin, T. (2004). Socioeconomic variation in attitudes to eating and weight in female adolescents. Health Psychology, 23(3), 275-282.

W est, P., \& Sweeting, H. (2004). Evidence on equalization in heal th in youth from the W est of Scotland. Social Science \& M edicine, 59(1), 13-27.

W est, P. (1997). Health inequalities in the early years: Is there equalization in youth? Social Science and M edicine, 44, 833-858. 